\title{
A Statistical User Simulation Technique for the Improvement of a Spoken Dialog System ${ }^{\star}$
}

\author{
Lluís F. Hurtado, David Griol, Emilio Sanchis, and Encarna Segarra \\ Departament de Sistemes Informàtics i Computació (DSIC) \\ Universitat Politècnica de València (UPV) \\ Camí de Vera s/n, 46022 València, Spain \\ \{lhurtado, dgriol, esanchis, esegarra\}@dsic.upv.es
}

\begin{abstract}
In this paper, we present a statistical approach for the automatic generation of dialogs by means of a user simulator. This technique can be used to generate dialogs with reduced effort, facilitating the evaluation and improvement of spoken dialog systems. In our approach for user simulation, the user answer is selected taking into account the history of the dialog and the last system turn, as well as the objective(s) set for the dialog. The user model is automatically learned from a training corpus that is labeled in terms of dialog acts. This methodology has been evaluated within the framework of the DIHANA project, whose goal is the design and development of a dialog system to access a railway information system using spontaneous speech in Spanish.
\end{abstract}

Keywords: spoken dialog systems, user simulation, dialog management, dialog system evaluation.

\section{Introduction}

A dialog system is a man-machine interface that is able to recognize and to understand a spoken input and to produce an oral output as answer. Different modules usually take part in order to carry out this final goal: they must recognize the pronounced words, understand their meaning, manage the dialog, make the error handling, access to the databases and generate the oral answer. Nowadays, diverse projects have developed systems to provide information and other services automatically; for example, information and booking of airplane and train trips [1] [2] [3] [4] and other types of information [5] [6] [7].

An important area of research within the framework of dialog systems is the development of techniques that facilitate the evaluation of these systems and the learning of an optimal strategy for dialog management. A technique that has attracted increasing interest in the last decade is based on the automatic generation of dialogs between the Dialog Manager (DM) and an additional module, called user simulator, which represents user interactions with the dialog system. The user simulator makes it possible to

\footnotetext{
* This work has been partially supported by the Spanish Government and FEDER under contract TIN2005-08660-C04-02, and by the Vicerrectorado de Innovación y Desarrollo of the Universidad Politécnica de Valencia under contract 4681.
} 
generate a great number of dialogs in a very simple way. Therefore, this technique reduces the time and effort that would be needed for the evaluation of a dialog system with real users each time the system is modified.

The construction of user models based on statistical methods has provided interesting and well-founded results in recent years and is currently a growing research area. The overall goal of techniques of this kind is to learn optimal strategies for dialog management from training data. A probabilistic user model can be trained from a corpus of human-computer dialogs to simulate user answers. Therefore, it can be used to learn a dialog strategy by means of its interaction with the DM. In the literature, there are several approaches that are related to the development of corpus-based methods for user simulation. These describe different alternatives for carrying out the evaluation of the techniques and for learning optimal management strategies and evaluating the dialog system [8] [9] [10] [11]. A summary of user simulation techniques for reinforcement learning of the dialog strategy can be found in [12].

We have developed different statistical approaches for the design of a DM [13] [14] and for user modeling [15]. The methodology that we present in this paper for developing a user simulator extends our work to model the system behavior, which is described in [14]. The user simulator is automatically learned from a training corpus that is labeled in terms of dialog acts. In our system, both DM and user behaviors are corpus-based.

The new user turn is selected by taking into account the information provided by the simulated user throughout the history of the dialog, the last system turn, and the objective(s) set for the dialog. The user turn, which is represented as dialog acts, is selected using the probability distribution provided by a neural network. With this methodology, an initial dialog corpus can be extended by increasing its variability and detecting dialog situations in which the DM does not provide an appropriate answer.

This approach has been used for the construction of a user simulator within the framework of the DIHANA project [16]. This project undertakes the design and development of a dialog system for access to an information system using spontaneous speech. The domain of the project is the query to an information system about railway timetables, fares, and services in Spanish.

Section 2 briefly presents the main characteristics of the dialog system developed for the DIHANA project. It also describes a corpus and the semantic and dialog-act labeling that is used for learning the user model. Section 3 presents the proposed user simulator. Section 4 and 5 present an evaluation of this approach and our conclusions.

\section{The System Architecture}

Within the framework of the DIHANA project, we have developed a mixed-initiative dialog system to access information systems using spontaneous speech [16]. We have built an architecture that is based on the client-server paradigm. The system consists of six modules: an automatic speech recognition (ASR) module, a natural language understanding (NLU) module, a dialog manager (DM), a database query manager, a natural language answer generator, and a text-to-speech converter.

We are currently using the CMU Sphinx-II system (cmusphinx.sourceforge.net) in our speech recognition module. For speech output, we have integrated the Festival 


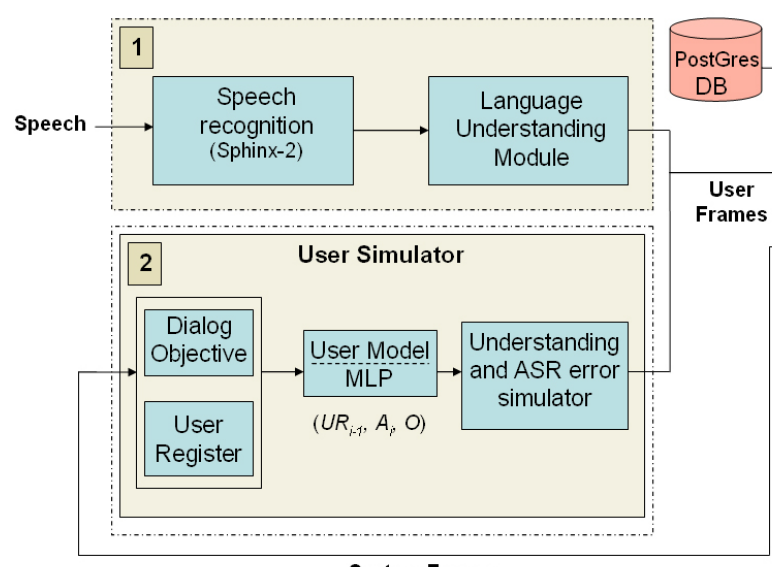

System Frames

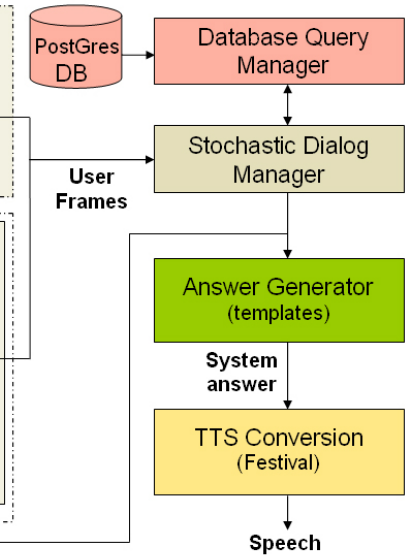

Speech

Fig. 1. Architecture of the DIHANA dialog system. [1] Interaction with real users. [2] Operation with the user simulator.

speech synthesis system (www.cstred.ac.uk/projects/festival). The specific information relative to our task is stored in a PostGres database using dynamic information extracted from the web.

Our dialog system has two operation modes. First, the system uses the ASR and the NLU modules for the normal interaction between the system and the real users. Second, the system allows the automatic acquisition of dialogs by means of the user simulator module. Figure 1 1shows the modular architecture of our system: (1) the interaction with real users and (2) the operation with the user simulator.

The behavior of the principal modules that make up the dialog system is based on statistical models that are learned from a dialog corpus that was acquired and labeled within the framework of the DIHANA project.

\subsection{The DIHANA Corpus}

A set of 900 dialogs (6,280 user turns) was acquired for the DIHANA project using the Wizard of $\mathrm{Oz}$ technique (WOz). Real ASR and NLU modules were used for the acquisition and the WOz played the role of the DM following a predefined strategy. Five files were stored for each acquired dialog: the output of the recognizer, the output of the understanding module, the answer (dialog act) generated by the system, the values of the attributes during the successive turns, and the queries made to the Database. This information is used to model the behavior of the system depending on the succession of dialog acts, the semantic representation of the user turn, and the values associated to the attributes (and their confidence scores).The characteristics of this corpus are shown in Figure 2

For the acquisition, a set of 300 scenarios were defined. These scenarios can be classified into two categories depending on the number of objectives. Type S1 defined only one objective for the dialog and Type S2 defined two objectives. 


\begin{tabular}{|l|r|}
\hline Number of users & 225 \\
\hline Number of dialogs/user & 4 \\
\hline Number of user turns & 6280 \\
\hline Average number of user turns/dialog & 7 \\
\hline Average number of words/user turn & 7.74 \\
\hline Vocabulary & 823 \\
\hline Duration of the recording (hours) & 10.8 \\
\hline
\end{tabular}

Fig. 2. Main characteristics of the DIHANA corpus

In order to learn statistical dialog models, the dialogs were labeled in terms of dialog acts. The user dialog acts correspond to the classical frame representation of the meaning of the utterance. In other words, one or more concepts represent the intention of the utterance, and a sequence of attribute-value pairs contains the information about the values given by the user. The Understanding Module takes the sentence supplied by the recognition process as input and generates one or more frames as output. In this task, we defined eight concepts and ten attributes. There are two kinds of concepts:

1. Task-dependent concepts: they represent the concepts the user can ask for, such as Hour, Price, Train-Type, Trip-Time and Services.

2. Task-independent concepts: they represent typical interactions in a dialog, such as Affirmation, Negation and Not-Understood.

The attributes are: Origin, Destination, Departure-Date, Arrival-Date, DepartureHour, Arrival-Hour, Class, Train-Type, Order-Number and Services.

The system turns were labeled using a set of three-level dialog acts. The first level describes the general acts of any dialog independently of the task. The second level represents the concepts involved in the turn and is specific to the task. The third level represents the values of the attributes given in the turn. The following labels were defined for the first level: Opening, Closing, Undefined, Not-Understood, Waiting, New-Query, Acceptance, Rejection, Question, Confirmation and Answer. The labels defined for the second and third level were the following: Departure-Hour, Arrival-Hour, Price, TrainType, Origin, Destination, Date, Order-Number, Number-Trains, Services, Class, TripType, Trip-Time and Nil. Each turn of the dialogs was labeled with one or more dialog acts. From this kind of detailed dialog act labeling and the values of attributes obtained during a dialog, it is straightforward to construct a sentence in natural language.

An example of the user/system labeling is shown below:

\section{User turn:}

I would like to know the timetables from Valencia to Madrid.

\section{Semantic representation:}

(Hour)

Origin: Valencia

Destination: Madrid 


\section{System turn:}

Do you want timetables to Madrid?

\section{Three-level labeling:}

(Confirmation:Arrival-Hour:Destination)

A more detailed description about the $\mathrm{WOz}$ acquisition and the corpus labeling can be found in [14].

\section{The User Simulator}

In our system, the user simulator replaces the functions performed by the ASR and the NLU modules. It generates frames in the same format defined for the output of the NLU module, i.e, in the format expected by the DM.

The methodology that we have developed for user simulation is based on the statistical modelization of the sequences of user and system dialog acts. As stated above, the user answers are generated taking into account the information provided by the simulator throughout the history of the dialog, the last system turn, and the objective(s) predefined for the dialog. A labeled corpus of dialogs is used to estimate the user model. The formal description of the proposed model extends the methodology proposed in DIHANA for dialog management:

Let $A_{i}$ be the output of the dialog system (the system answer) at time $i$, expressed in terms of dialog acts. Let $U_{i}$ be the semantic representation of the user turn. We represent a dialog as a sequence of pairs (system-turn, user-turn):

$$
\left(A_{1}, U_{1}\right), \cdots,\left(A_{i}, U_{i}\right), \cdots,\left(A_{n}, U_{n}\right)
$$

where $A_{1}$ is the greeting turn of the system (the first turn of the dialog), and $U_{n}$ is the last user turn. We refer to a pair $\left(A_{i}, U_{i}\right)$ as $S_{i}$, the state of the dialog sequence at time $i$.

Given this representation, the objective of the user simulator at time $i$ is to find an appropriate user answer $U_{i}$. This selection, which is a local process for each time $i$, takes into account the sequence of dialog states that precede time $i$, the system answer at time $i$, and the objective of the dialog $\mathcal{O}$. If the most probable user answer $U_{i}$ is selected at each time $i$, the selection is made using the following maximization:

$$
\hat{U}_{i}=\underset{U_{i} \in \mathcal{U}}{\operatorname{argmax}} P\left(U_{i} \mid S_{1}, \cdots, S_{i-1}, A_{i}, \mathcal{O}\right)
$$

where set $\mathcal{U}$ contains all the possible user answers.

As the number of possible sequences of states is very large, we establish a partition in this space (i.e., in the history of the dialog preceding time $i$ ).

Let $U R_{i}$ be the user register at time $i$. The user register is defined as a data structure that contains the information about concepts and attribute values provided by the user throughout the previous history of the dialog. The information contained in $U R_{i}$ is a summary of the information provided by the sequence $S_{1}, \ldots, S_{i-1}$. Different state sequences can lead to the same $U R$.

The partition that we establish in this space is based on the assumption that two different sequences of states are equivalent if they lead to the same $U R$. This assumption 
provides a great reduction in the number of different histories in the dialogs at the expense of a loss in the chronological information. However, we do not consider the order in which the information is supplied by the user to be a relevant factor in determining the next user turn $U_{i}$.

After applying the above considerations and establishing the equivalence relations in the histories of the dialogs, the selection of the best $U_{i}$ is given by:

$$
\hat{U}_{i}=\underset{U_{i} \in \mathcal{U}}{\operatorname{argmax}} P\left(U_{i} \mid U R_{i-1}, A_{i}, \mathcal{O}\right)
$$

In our previous work on dialog management, we proposed the use of a multilayer perceptron (MLP) to obtain the system answer. In this work, we also propose using the MLP to make the assignation of a user turn. The input layer receives the current situation of the dialog, which is represented by the term $\left(U R_{i-1}, A_{i}, \mathcal{O}\right)$ in Equation 1 . The values of the output layer can be viewed as the a posteriori probability of selecting the different user answers defined for the simulator given the current situation of the dia$\log$. The choice of the most probable user answer of this probability distribution leads to Equation 1 In this case, the user simulator will always generate the same answer for the same situation of the dialog. Since we want to provide the user simulator with a richer variability of behaviors, we base our choice on the probability distribution supplied by the MLP on all the feasible user answers.

\subsection{Codification Defined for the MLP Classifier}

For the DIHANA task, the $U R$ is a sequence of 15 fields that correspond to the five concepts (Hour, Price, Train-Type, Trip-Time, and Services) and ten attributes (Origin, Destination, Departure-Date, Arrival-Date, Departure-Hour, Arrival-Hour, Class, Train-Type, Order-Number, and Services) defined for our task. For the user simulator to determine the next user turn, we have assumed that the exact values of the attributes are not significant. Even though these values are important for accessing the database and for constructing the output sentences of the system, the only information necessary to determine the next user action is the presence or absence of concepts and attributes. Therefore, the information we used from the $U R$ is a codification of this data in terms of two values, $\{0,1\}$, for each field in the $U R$ according to the following criteria: $\mathbf{0}$ if the concept is not activated, or the value of the attribute is not given; $\mathbf{1}$ if the concept or attribute is activated.

For the DM of the DIHANA task, we have defined a total of 51 possible system answers taking into account the different combinations of three-level labeling. Thus, the system answer is modeled using a variable with 51 bits.

\subsection{The Error Simulator}

Our corpus includes information about the errors that were introduced by the ASR and the NLU modules during the acquisition. This information also includes confidence measures, which are used by the DM to evaluate the reliability of the concepts and attributes generated by the NLU module. 
An error simulator module has been designed to perform error generation and the addition of confidence measures in accordance with an analysis made of our corpus. This information modifies the frames generated by the user simulator. Experimentally, we have detected 2.7 errors per dialog. This value can be modified to adapt the error simulator module to the operation of any ASR and NLU modules. As future work, we want to make a more detailed study of the errors introduced in our corpus.

\section{Evaluation}

To carry out the evaluation of the simulation process, 50,000 dialogs of each one of the two types of scenarios defined (Type S1 and Type S2) were generated.

Two criteria were defined for closing the dialog. The first criterion consists of finalizing the dialog when the number of system turns exceeds a threshold obtained experimentally. The second criterion is applied to generate a user request to close the dialog when the manager has provided the set of information defined for the objective(s) of the dialog. The successful dialogs are those that end when the second criterion is applied.

We defined five measures for the evaluation of the simulated dialogs: the number of successful dialogs (SD), the average number of turns per dialog (NT), the number of different successful dialogs (DD), the number of turns of the shortest dialog (TS), and the number of simulated dialogs that are contained in our initial corpus (CD). Using these measures, we tried to evaluate the success of the simulated dialogs as well as its efficiency and variability with regard to the different objectives.

Table 1 shows the values of the different measures obtained for the simulated corpus of 100,000 dialogs. Taking the two types of scenarios into account, the simulator generated a total of 22,614 dialogs that achieved their objectives $(22.6 \%)$. The number of simulated dialogs that were initially contained in the WOz corpus, $\mathrm{CD}$, corresponds to only a small partition of these dialogs (1.2\%). It can be viewed that Type S2 dialogs are more complicated to simulate due to the two objectives must be satisfied.

Table 1. Evaluation of the simulated corpus

\begin{tabular}{|r|r|r|r|r|r|}
\hline Type & SD & NT & DD & TS & CD \\
\hline S1 & 18,400 & 10.4 & 11,550 & 5 & 224 \\
S2 & 4,214 & 12.1 & 3,833 & 7 & 42 \\
\hline
\end{tabular}

First, we evaluated the behavior of the original DM that was learned using the training corpus (obtained by WOz). Then, we evaluated its evolution when the successful simulated dialogs were incorporated to the training corpus. A new DM model was learned each time a new set of simulated dialogs was generated. For this evaluation, we used a test partition that was extracted from the DIHANA corpus ( $20 \%$ of the samples). We considered four measures: the number of unseen situations (\#unseen), i.e. the dialog situations that are present in the test partition but not in the corpus used for learning the DM; the number of answers provided by the DM that would cause the failure of the dialog (\#error); the percentage of answers provided by the DM that exactly 
follow the strategy defined for the WOz to acquire the training corpus (\%strategy); and the percentage of answers provided by the DM that are coherent with the current state of the dialog although they do not follow the original strategy defined for the $\mathrm{WOz}$ (\%coherent).

Figure 3 and Figure 4 respectively show how \#unseen and \#error decreased when the training corpus was enriched by adding the simulated dialogs, which is the expected behavior. These measures continued to decrease until 60,000 dialogs were simulated.



Fig. 3. Evolution of the \#unseen with regard to the incorporation of new simulated dialogs

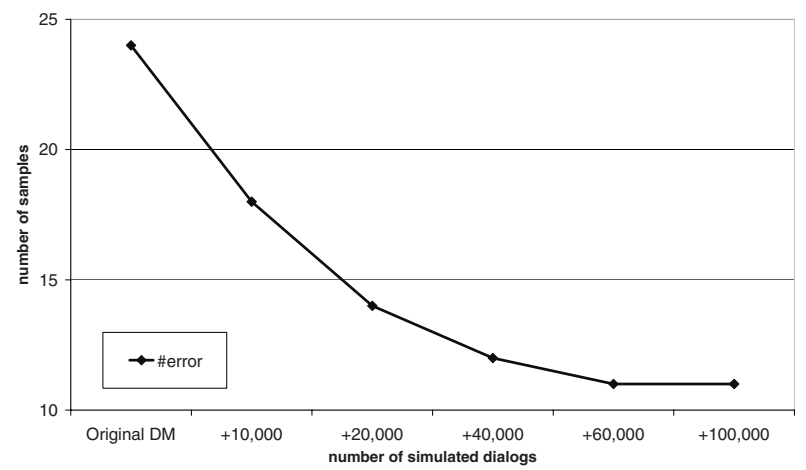

Fig. 4. Evolution of the \#error with regard to the incorporation of new simulated dialogs

Figure 5 shows the evolution of \%strategy and \%coherent. It can be observed that the DM improved the generation of coherent answers when the new dialogs were incorporated. In addition, the number of coherent answers that are different from those defined in the WOz strategy was increased. In other words, the original strategy was modified, thereby allowing the DM to tackle new situations and generate new coherent answers. 


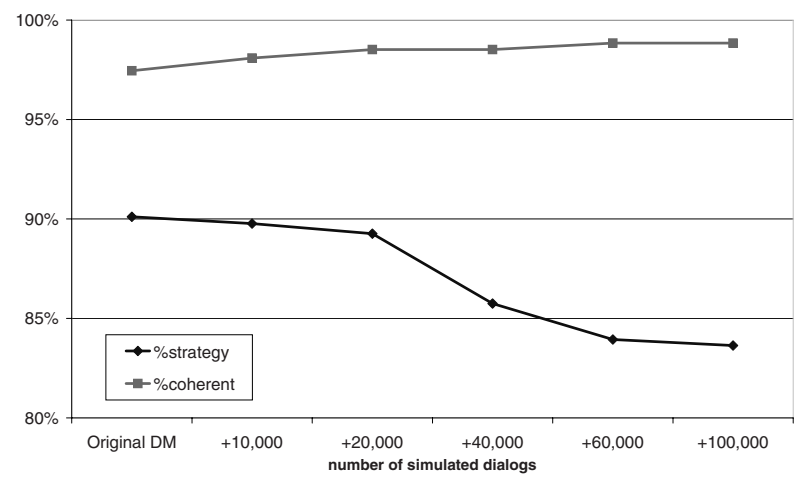

Fig. 5. Evolution of the \%strategy and \%coherent with regard to the incorporation of new simulated dialogs

\section{Conclusions}

In this paper, we have presented a corpus-based approach for the development of a user simulator. The proposed methodology allows the generation of new dialogs with little effort. We have described an evaluation of this methodology within the framework of a dialog system, in which both the DM and the user simulator are statistically modeled from a data corpus. The definition of the user register allows the user simulator to take into account the complete history of the dialog in order to generate the next user turn.

The results of the evaluation demonstrate that the coverage of the DM is increased by incorporating the successful simulated dialogs and that the number of unseen situations can be reduced. A study of the evolution of the strategy followed by the DM has also been carried out. This study shows how the DM modifies its strategy by detecting new correct answers that were not defined in the initial strategy. As future work, we plan to apply this technique to a new domain, the booking of sports installations, to evaluate the task-portability.

\section{References}

1. Seneff, S., Polifroni, J.: Dialogue management in the Mercury flight reservation system. In: Proc. ANLP-NAACL 2000, Satellite Workshop pp. 1-6 (2000)

2. Lamel, L., Rosset, S., Gauvain, J., Bennacef, S., Garnier-Rizet, M., Prouts, B.: The LIMSI ARISE System. Speech Communication 4(31), 339-353 (2000)

3. Rudnicky, A., Thayer, E., Constantinides, P., Tchou, C., Shern, R., Lenzo, K., Xu, W., Oh, A.: Creating natural dialogs in the Carnegie Mellon Communicator system. Proceedings of Eurospeech 1(4), 1531-1534 (1999)

4. Ward, W., Pellom, B.: The CU Communicator system. In: Proc. of ASRU-IEEE Workshop, Keystone, Colorado (USA) (1999)

5. Bohus, D., Grau, S., Huggins-Daines, D., Keri, V., Krishna, G., Kumar, R., Raux, A., Tomko, S.: Conquest - an open-source dialog system for conferences. In: Proc. HLT-NAACL 2007, Rochester, NY (2007) 
6. Litman, D., Singh, S., Kearns, M., Walker, M.: Njfun: a reinforcement learning spoken dialogue system. In: Proceedings of the ANLP/NAACL 2000 Workshop on Conversational Systems, Seattle, USA, Association for Computational Linguistics pp. 17-20 (2000)

7. Zue, V., Seneff, S., Glass, J., Polifroni, J., Pao, C., Hazen, T., Hetherington, L.: Jupiter: A telephone-based conversational interface for weather information. IEEE Trans. on Speech and Audio Proc. 8(1), 85-96 (2000)

8. Georgila, K., Henderson, J., Lemon, O.: User Simulation for Spoken Dialogue Systems: Learning and Evaluation. In: Proc. of Interspeech 2006-ICSLP, Pittsburgh, pp. 1065-1068 (2006)

9. Pietquin, O., Dutoit, T.: A probabilistic framework for dialog simulation and optimal strategy learning. IEEE Transactions on Speech and Audio Processing 14, 589-599 (2005)

10. Scheffler, K., Young, S.: Automatic learning of dialogue strategy using dialogue simulation and reinforcement learning. In: Proc. Human Language Technology, San Diego, pp. 12-18 (2002)

11. Cuayáhuitl, H., Renals, S., Lemon, O., Shimodaira, H.: Learning multi-goal dialogue strategies using reinforcement learning with reduced state-action spaces. In: Proc. of InterSpeech'06-ICSLP, Pittsburgh, pp. 469-472 (2006)

12. Schatzmann, J., Weilhammer, K., Stuttle, M., Young, S.: A Survey of Statistical User Simulation Techniques for Reinforcement-Learning of Dialogue Management Strategies. Knowledge Engineering Review 21(2), 97-126 (2006)

13. Griol, D., Hurtado, L., Segarra, E., Sanchis, E.: Managing unseen situations in a stochastic dialog model. In: Proc. of AAAI Workshop Stadistical and Empirical Approaches for Spoken Dialogue Systems, Boston, pp. 25-30 (2006)

14. Hurtado, L.F., Griol, D., Segarra, E., Sanchis, E.: A stochastic approach for dialog management based on neural networks. In: Proc. of Interspeech 2006-ICSLP, Pittsburgh, pp. 49-52 (2006)

15. Torres, F., Sanchis, E., Segarra, E.: Learning of stochastic dialog models through a dialog simulation technique. In: Proc. of Eurospeech 2005, Lisbon, pp. 817-820 (2005)

16. Griol, D., Torres, F., Hurtado, L., Grau, S., García, F., Sanchis, E., Segarra, E.: A dialog system for the DIHANA Project. In: Proc. of SPECOM 2006, S. Petersburgh (2006) 